\title{
Energy and Link Measurements for Mobile Phones using IEEE802.11b/g
}

\author{
Morten V. Pedersen and Gian Paolo Perrucci and Frank H.P. Fitzek and Torben Larsen \\ Aalborg University, Denmark, e-mail: [mvpe|gpp|ff|tl]@es.aau.dk
}

\begin{abstract}
In this paper we investigate the energy consumption and achievable data rates of commercially available mobiles phones using WLAN IEEE802.11bg for mobile peer to peer networking. In contrast to numerous data rate measurements for WLAN, not much attention was paid for the energy consumption using WLAN on mobile phones. The knowledge of the actual energy consumption is useful for any future protocol design. Therefore we present a solid measurement campaign looking into the energy spent in sending, receiving, and idle mode for unicast and broadcast traffic. We introduce our measurement testbed as well as the tools that have been used or implemented throughout this work.
\end{abstract}

\section{INTRODUCTION AND MOTIVATION}

The full degree of freedom in mobile systems is based on the usage of batteries, which capacity is in general limited and for sure not keeping pace as the mobile devices are crammed up with new functionalities. The discrepancy of Moore's law, offering twice the processing power every second year, and the development in batteries, which did not even double over the last decade, makes a shift in researchers' way of designing networks, protocols, and the mobile device itself. The bottleneck to take care of in the designing process of mobile systems is not only the wireless data rate anymore, but also the energy limitation as the customers will ask for new services, realized through larger data rates, and longer standby or operational times of their mobile devices.

In this paper we look into the energy and link characteristic of WLAN enabled mobile phones forming mobile peer to peer networks. We use commercially available mobile phones, in this case the Nokia N95. The investigation in energy consumption is the ground work for our work on mobile peer to peer networks as well as cooperative wireless networks [1], [2], but is certainly interesting for other researchers as well as it will provide realistic values for their analysis of wireless networks. As we look into mobile peer to peer networks we are mostly interested in the link characteristics among mobile phones without the usage of any access point.

The state of the art in this field can be described as such, that a lot of work has been done for WLAN measurements [3]. Most of the work is focusing on the data rate only. Other researcher have been looking already in the energy issues [4], [5], [6] some years ago. Nevertheless this paper is different from the state of the art as we will carry out the WLAN measurements between mobile phones using the adhoc mode with the newest WLAN technology integrated in mobile phones.

\section{Measurement Testbed}

In the following we explain the measurement testbed. First the setup of the measurement campaign and the used components are introduced. As all measurements are carried out by mobile phones, we explain which tools were used and which applications had to be implemented from the scratch.

\section{A. Setup and Components}

Our measurement campaign was carried out at Aalborg University, Denmark. The measurement location is a public space for students and employees. For the measurements we used commercially available mobile phones, namely Nokia N95 equipped with WLAN IEEE802.11bg. As given in Figure 1 we place the phones at certain points in a corridor equally spaced with 3 meters apart from each other. As the main point of interest is in the communication characteristics between mobile devices, no access point was used for our campaign. Nevertheless several other WLAN access points were active and causing interference while we did our measurements.

The mobile phone in position 0 was selected to send packets to the other mobile phones. That could be realized through unicast or broadcast. In the unicast scenario, the sending node sends all packets to one other dedicated mobile phone. On the MAC layer each packet is acknowledged by the receiving mobile phone in case of a successful transmission. In case of error-prone communication, no acknowledgment will be send and the sending mobile phone will repeat the transmission a certain number of times. In the broadcast scenario multiple mobile devices (position 1-f) are receiving from the sending mobile device (position 0). No MAC acknowledgments are exchanged in this case and higher loss rates must therefore be expected.

\section{B. Methodology}

To conduct the the measurement campaign is was necessary to develop a number of different tools, these will be described in the following.

1) WLANTester: In order to carry out the measurements we have implemented a Symbian application for S60 3rd edition that is able to send or receive packets over IEEE802.11 called WLANTester (see Figure 3). The sending application creates packets of size $S$ and conveys them to one or multiple receivers every $t$ milli-seconds using unicast or broadcast, respectively. The packets sent include a packet counter, which is used at the receiving mobile device to log successful and not successful packet transmissions. 
TABLE I

WLAN INTERFERENCE ENVIRONMENT

\begin{tabular}{llllrlrrr}
\hline Address & ESSID & Protocol & Mode & Channel & Encryp. & Bit Rate & Extra RSSI & Extra Last beacon \\
\hline \hline 00:0E:84:26:1D:30 & AAU & IEEE $802.11 \mathrm{bg}$ & Master & 1 & off & $54 \mathrm{Mb} / \mathrm{s}$ & $-55 \mathrm{dBm}$ & $12 \mathrm{~ms} \mathrm{ago}$ \\
\hline 00:0E:84:2E:80:C0 & AAU & IEEE 802.11bg & Master & 2 & off & $54 \mathrm{Mb} / \mathrm{s}$ & $-61 \mathrm{dBm}$ & $11 \mathrm{~ms} \mathrm{ago}$ \\
\hline 00:17:3F:5C:40:15 & belkin54g & IEEE 802.11bg & Master & 1 & off & $54 \mathrm{Mb} / \mathrm{s}$ & $-60 \mathrm{dBm}$ & $16 \mathrm{~ms} \mathrm{ago}$ \\
\hline 00:14:C2:B8:86:B1 & Thyguest & IEEE $802.11 \mathrm{bg}$ & Master & 7 & on & $54 \mathrm{Mb} / \mathrm{s}$ & $-74 \mathrm{dBm}$ & $4 \mathrm{~ms} \mathrm{ago}$ \\
\hline 00:1B:11:E7:5B:97 & Holtsmark & IEEE 802.11bg & Master & 7 & on & $54 \mathrm{Mb} / \mathrm{s}$ & $-63 \mathrm{dBm}$ & $6 \mathrm{~ms} \mathrm{ago}$ \\
\hline 00:0E:38:A8:E6:30 & AAU & IEEE $802.11 \mathrm{bg}$ & Master & 11 & off & $54 \mathrm{Mb} / \mathrm{s}$ & $-60 \mathrm{dBm}$ & $2 \mathrm{~ms} \mathrm{ago}$ \\
\hline
\end{tabular}

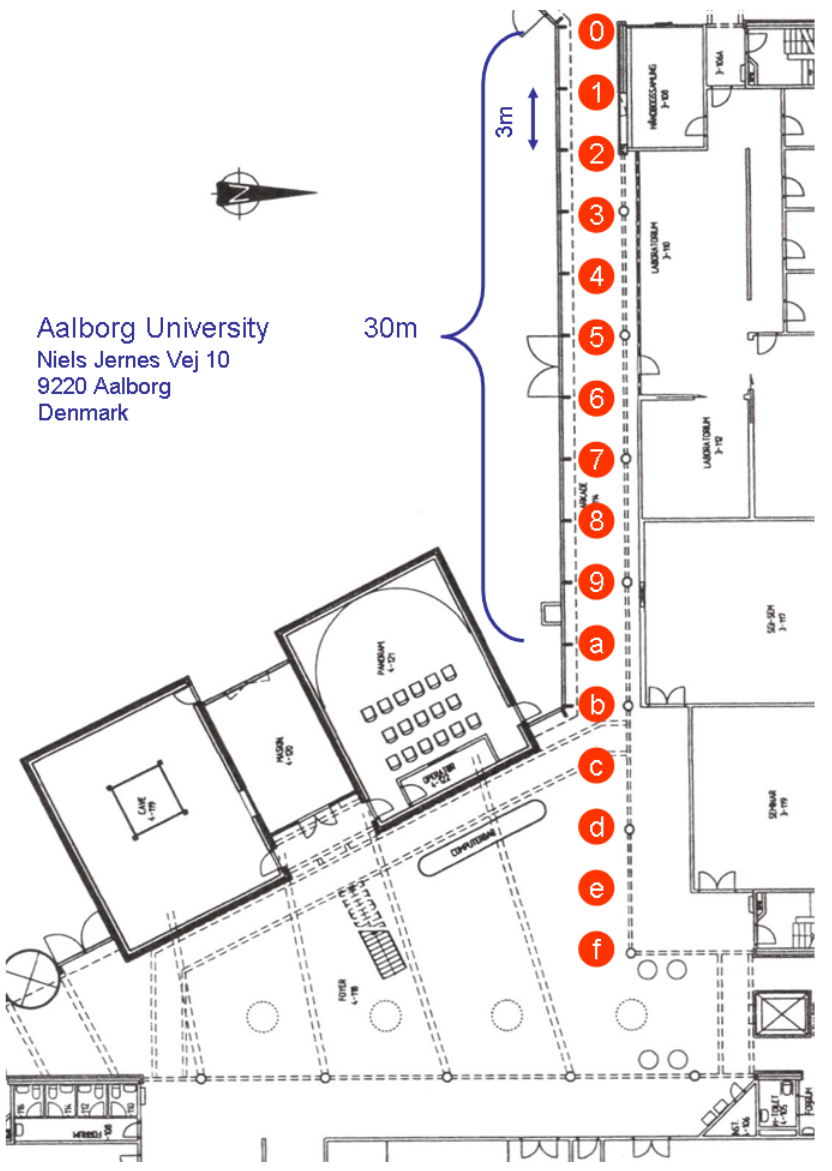

Fig. 1. Measurement campaign at Aalborg University - Niels Jernes Vej 10. The red dots shown the placement of the mobile phones. Each measurement point is equally spaced 3 meters apart.

In the application the following parameters could be configured (see Figure 4:

- packet size: The actual packet size in bytes that should be send out or received.

- number of packets: The number of packets that will be send or received within one test.

- number of tests: The number of tests carried out within one measurement campaign.

- inter packet time: The time between two packets.

- inter test time: The time between two tests.

The WLANTester application together with some small offline scripts were monitoring:

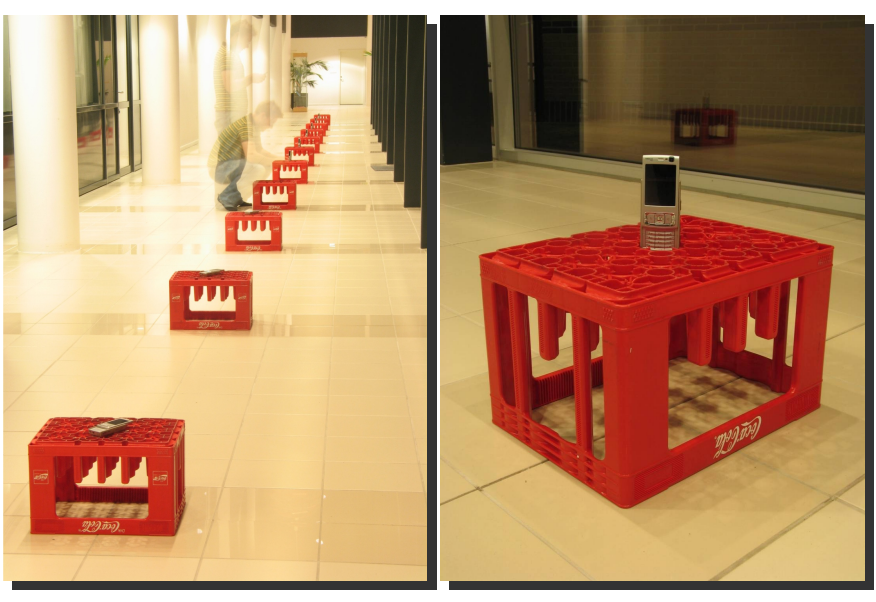

Fig. 2. Photo of the measurement campaign.

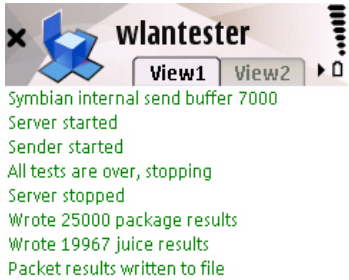

Options

Fig. 3. Screenshot of the WLANTester Fig. 4. Screenshot of the settings application.

- data rate: Counting the number of send or successfully received packets, the data rate was calculated.

- packet loss probability: The packet loss probability was calculated by the number of lost packets over the overall send packets.

Furthermore the WLANTester application controlled the Nokia Energy Profiler application to profile the power consumption while sending/receiving and idle.

2) Nokia Energy Profiler: The Nokia Energy Profiler application was used to measure the power consumption in the different sending and receiving phases directly on the mobile 
device. The Nokia Energy Profiler application was delivered by Nokia and is controlled from the WLANTester application (measurements can be stopped, started, paused, etc).

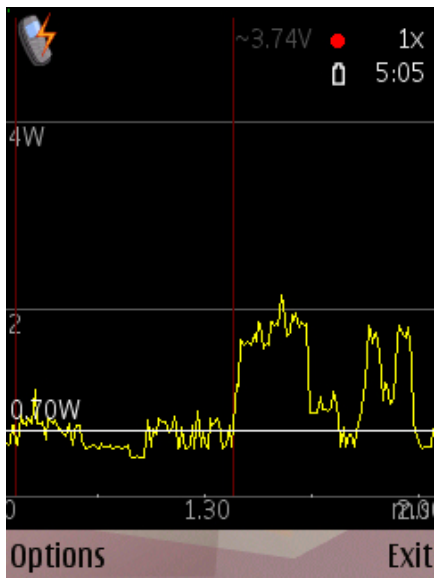

Fig. 5. Screenshot of the Nokia Energy Profiler application.

The advantage of the Nokia Energy Profiler application is that it can be installed on many phones at the same time.

3) Mobile Phone Settings: To ensure that all mobile devices configured in the same way the following settings was used on all mobile devices.

a) Display: The display settings can be found under: Tools $\longrightarrow$ Settings $\longrightarrow$ General $\longrightarrow$ Personalisation $\longrightarrow$ Display where the following settings where used.

- Light sensor: Set minimum.

- Power saver time-out: $5 \mathrm{sec}$

- Light time-out: $5 \mathrm{sec}$

b) Bluetooth: The Bluetooth module was turn off for all kind of measurements and never used.

c) Access Points: All the phones used an ad-hoc internet access point (internet access point, refers to an available connection on the mobile devices) for the WLAN tests, the access point configuration settings can be found under: Tools $\longrightarrow$ Settings $\longrightarrow$ Connection $\longrightarrow$ Access points. The following settings where used for the access point:

- Connection Name: TestWLAN

- Data bearer: Wireless LAN

- WLAN network name: TestWLAN

- WLAN network mode: Ad hoc

- Ad Hoc channel: 1

In addition to these settings the access point was defined to used static IP addresses, these where configured using the following settings (found under Options $\longrightarrow$ Advanced Settings $\longrightarrow$ IPv4 Settings):

- Phone IP address: 10.0.0.x (where $\mathrm{x}$ is the phone id, e.g. N95-006 should have 10.0.0.6 as IP)

- Subnet mask: 255.255.255.0

- Default gateway: 10.0.0.254
4) Measurement Reports: All mobile phones are reporting for each measurement the packet size, distance, power consumption etc. The reported values are evaluated offline and contain a plot for the application layer data rate, the power, the elapsed time, and the packet loss for each test (small round circles) and the mean value (solid line). The power plot is different as it carries two values, namely that for the idle and the receiving or sending mode. An example of such a report is given for the receiver with packet size 1000 byte, $12 \mathrm{~m}$ distance using the broadcast mode in as given in Figure 6. Those individual reports are then combined to $3 \mathrm{D}$ plots giving an overview of the relationships between different factors as shown in the following sections.
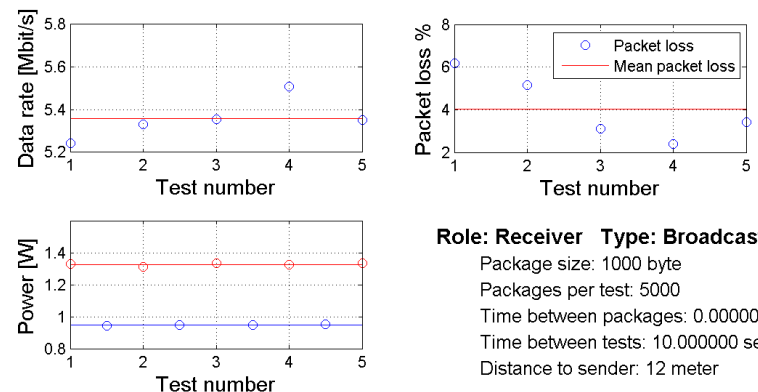

Role: Receiver Type: Broadcast Package size: 1000 byte Packages per test: 5000 Time between packages: $0.000000 \mathrm{sec}$ Time between tests: $10.000000 \mathrm{sec}$ Distance to sender: 12 meter

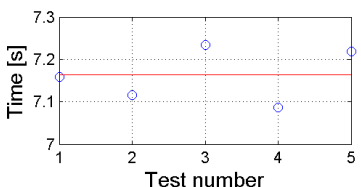
Test Results:

Mean package loss: $4.044000 \%$ Mean good-put: $5.357506[\mathrm{Mbit} / \mathrm{s}]$ Mean power: $1.326942[\mathrm{~W}]$ Mean listen power: $0.948282[\mathrm{~W}]$ Mean time: 7.163125 [s]

Fig. 6. Individual report of a mobile phone for each measurement.

\section{ENERGY CONSUMPTION AND DATA RATE FOR THE WLAN802.11BG}

We are interested in the energy consumption and the data rate for unicast and broadcast transmissions. The following notation is used throughout the paper:

- data rate: The data rate is calculated by sending 5000 packets per test, multiply it with the packet size and divide it by the mean time needed to run one test.

- energy: The energy is calculated by the power levels in the different states (sending/receiving/idle) multiplied by the time of a given state.

- packet loss: The packet loss is calculated by all non successfully received packets divided by the overall send packets.

- time: The time we measure referrers to a single test sending 5000 packets.

\section{A. Unicast Measurements}

In the unicast scenario we have one sending node and one receiving node placed at certain distances between $3 \mathrm{~m}$ and $30 \mathrm{~m}$. Different packet sizes were used between 100 byte and 1250 byte. 
In Figure 7 the data rate versus packet size and distance is given for the receiver. With an increased packet size the data rate increases as well until using more than 1250 byte. Larger values for the packet size (next size 1500 byte) seems to lead to fragmentation and therefore smaller data rates. In case of small distances between sender and receiver the data rate is larger due to rate adaptation. With larger distances the data rates are more or less stable.

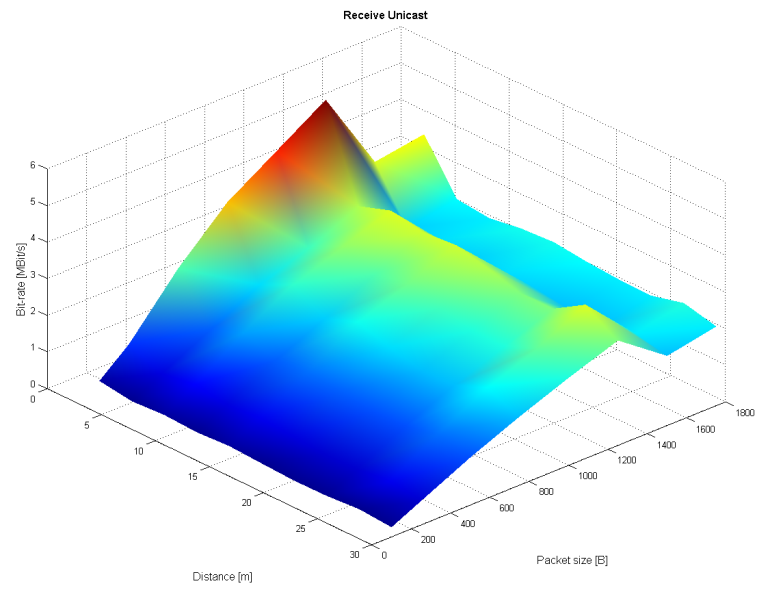

Fig. 7. Data rate at the receiver versus packet size and distance (between sender and receiver).

In Figure 8 and 9 the energy per byte is given for sender and receiver. Larger energy per byte values for smaller packets is due to the MAC and PHY overhead. For packets that get fragmented the energy level slightly increases.

In Figure 10 the packet loss for the receiver is given. The losses are more or less randomly distributed with slightly larger values for shorter distances. We have no full explanation for that, but this could be to the data rate adaptation, where the sender tries to switch to the next higher modulation/coding level, which may fail from time to time.

In Figure 11 the mean time per test is given. The common characteristic is that for packet sizes needing fragmentation, the mean time increases significantly. This is also the reason for the smaller data rates at the same area (Figure 7).

The measurements done for the unicast scenario is summarized for the power values, data rates, and loss rates in Table II.

TABLE II

POWER LEVELS USEd IN UNICAST - 1000 Bytes

\begin{tabular}{lrrr}
\hline state & power value [W] & data rate [Mbps] & losses [\%] \\
\hline sending @ 3m & 1.645 & 4.781 & - \\
sending @ 30m & 1.674 & 2.387 & - \\
receiving @ 3m & 1.449 & 4.745 & 0.792 \\
receiving @ 30m & 1.329 & 2.392 & $*$ \\
idle & 1.027 & - & - \\
sleep & 0.04 & - & - \\
\hline
\end{tabular}

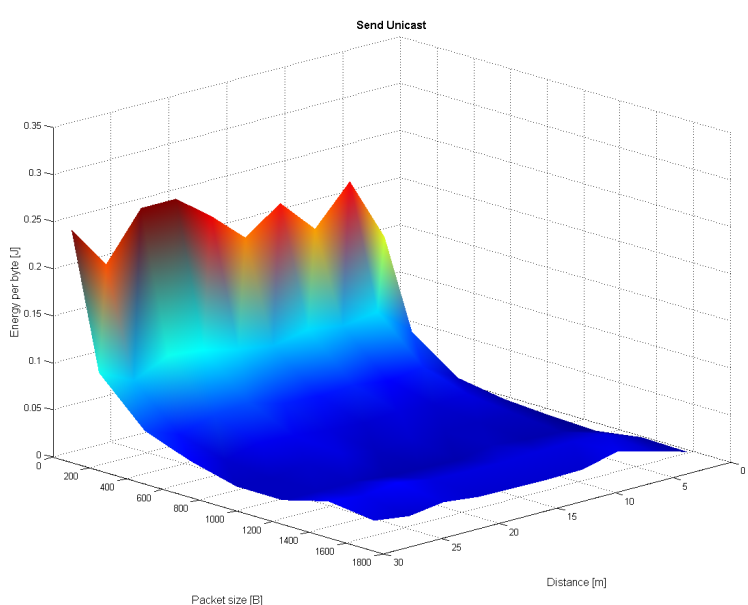

Fig. 8. Energy per byte at the sender versus packet size and distance (between sender and receiver).

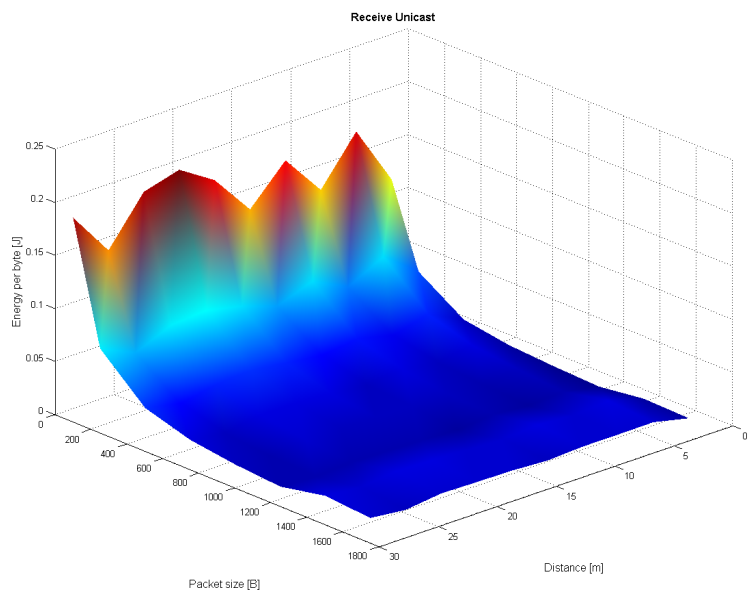

Fig. 9. Energy per byte at the receiver versus packet size and distance (between sender and receiver).

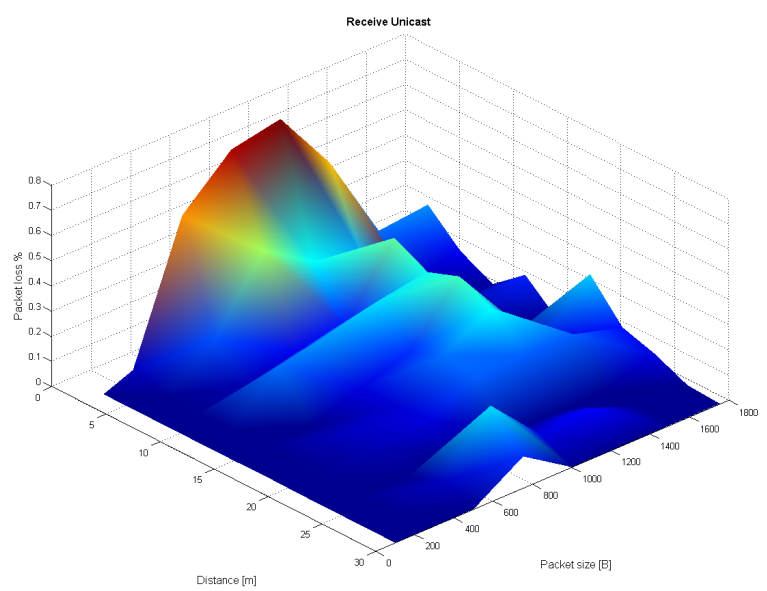

Fig. 10. Packet loss at the receiver versus packet size and distance (between sender and receiver) 


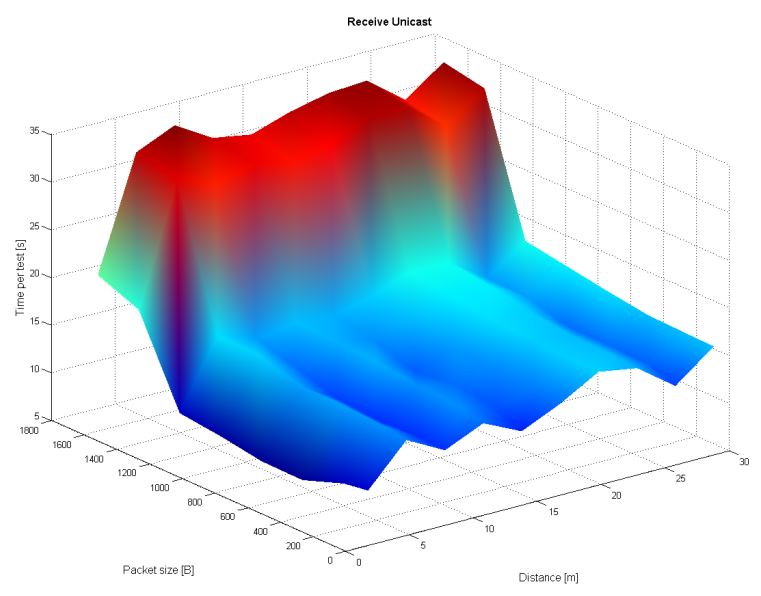

Fig. 11. Mean time per test at the receiver versus packet size and distance (between sender and receiver).

\section{B. Broadcast Measurements}

In the broadcast scenario we have one sending node and a number of receiving nodes placed at certain distance between $3 \mathrm{~m}$ and $30 \mathrm{~m}$. Different packet sizes were used between 100 byte and 1250 byte.

In Figure 12 the data rate at the receiver is given versus the packet size and the distance. By increasing the packet size we increase the MAC layer efficiency, by spreading the per-packet overhead over more bytes and imposing less contention in the channel. But due to losses on the wireless link the data rate decreases slightly with larger distances.

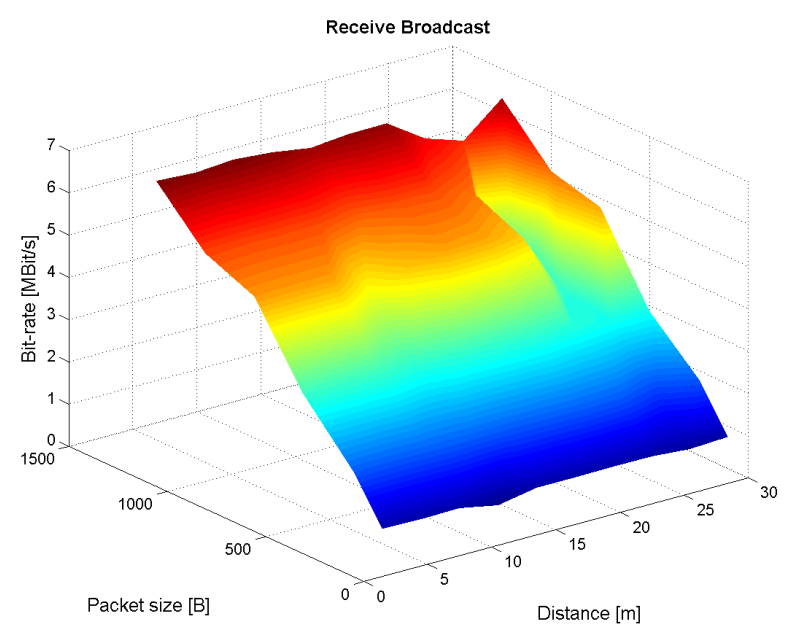

Fig. 12. Data rate at the receiver versus packet size and distance (between sender and receiver).

In Figure 13 and 14 the energy per byte is given over packet size and distance. In both figures a significant increase of energy per byte can be seen for small packets. This is due to the MAC and PHY overhead. The impact of the MAC and
PHY overhead vanishes with larger packet sizes. On receiver side we recognize smaller changes in the energy per byte due to packet losses. Packet losses lead to less energy consumption as less signal processing is done afterwards.

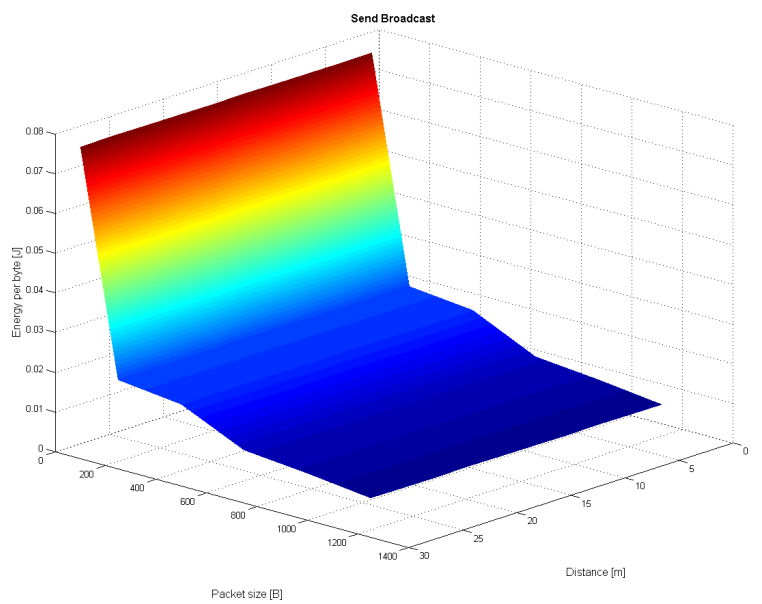

Fig. 13. Energy per byte at the sender versus packet size and distance (between sender and receiver).

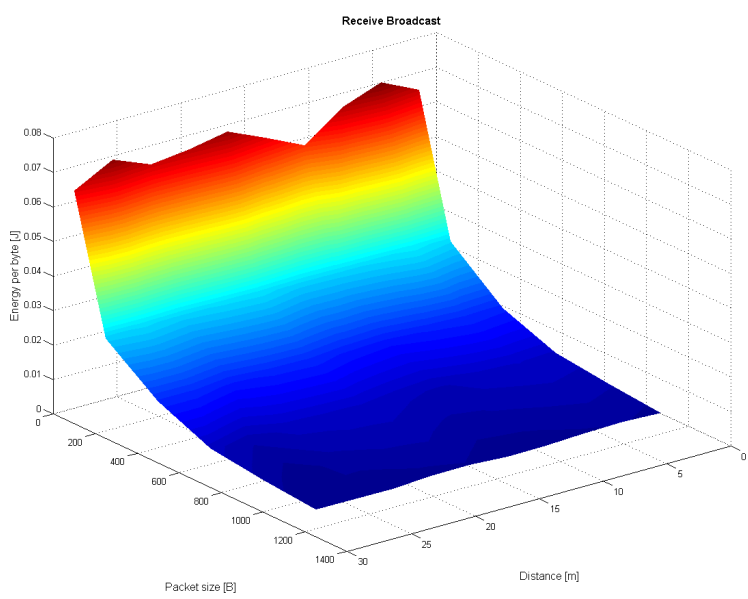

Fig. 14. Energy per byte at the receiver versus packet size and distance (between sender and receiver).

In Figure 15 the packet loss is given versus packet size and distance. On receiver side losses occur and with higher probability with larger distances. Note, the high peak at $30 \mathrm{~m}$ and 1250 byte is due to an error-prone measurement.

In Figure 16 the mean time needed to complete a full test sending 5000 packets has been depicted. In general, the larger the packet size the longer will take the measurements. The dip in the surface can be explained by data rate adaptation or a transient improvement of the channel conditions.

As for the unicast, Table III presents the power values, the data rate and losses.

\section{CONCLUSION}

In this paper we have presented energy and link measurements among mobile phones as used for mobile peer to peer 


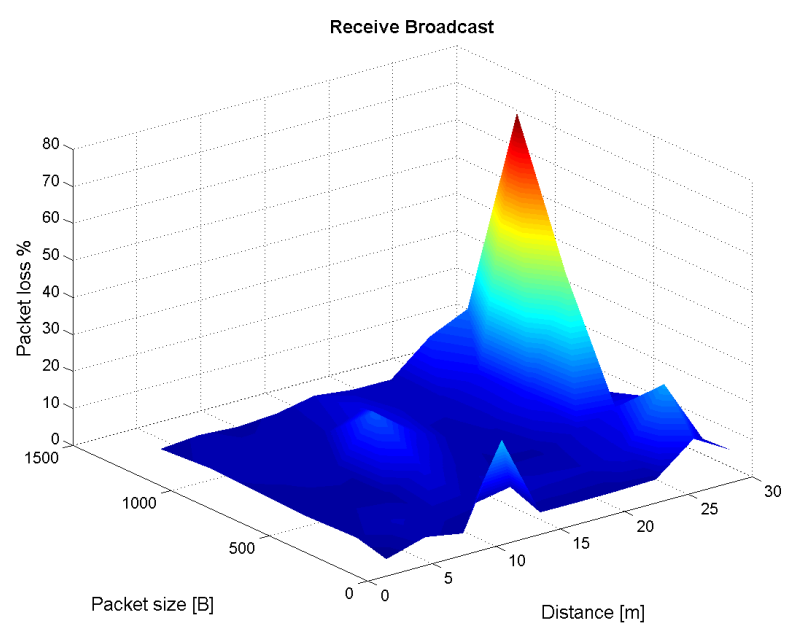

Fig. 15. Packet loss at the receiver versus packet size and distance (between sender and receiver).

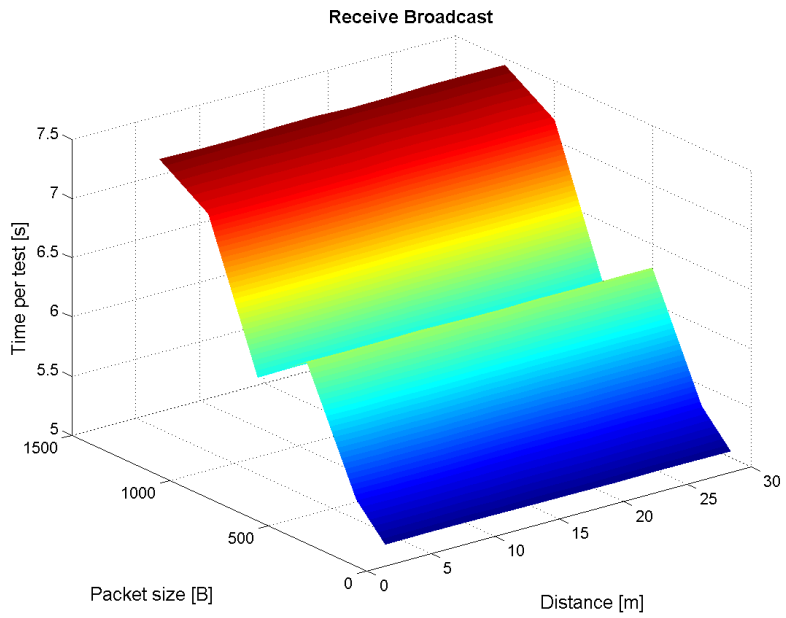

Fig. 16. Mean time per test at the receiver versus packet size and distance (between sender and receiver). networks using WLAN IEEE802.11. The results of this paper show the actual power levels in different transmission states such as sending, receiving, and idle. Furthermore achievable data rates and packet losses are given for unicast and broadcast transmissions. The results presented could be used by other researchers as input for their analysis and simulation of wireless communication networks.

\section{ACKNOWLEDGMENT}

We would like to thank Nokia for providing technical support as well as mobile phones to carry out the measurement campaign. Special thanks to Mika Kuulusa, Gerard Bosch, Harri Pennanen, Nina Tammelin, and Per Moeller from Nokia. This work was partially financed by the X3MP project granted by Danish Ministry of Science, Technology and Innovation.

\section{REFERENCES}

[1] F.H.P. Fitzek and M. Katz, Eds., Cognitive Wireless Networks: Concepts, Methodologies and Visions Inspiring the Age of Enlightenment of Wireless Communications, ISBN 978-1-4020-5978-0. Springer, 2007.

[2] F.H.P. Fitzek and M. Katz, Eds., Cooperation in Wireless Networks: Principles and Applications - Real Egoistic Behavior is to Cooperate!, ISBN 1-4020-4710-X. Springer, April 2006.

[3] Oliver Wellnitz, "Wlan 802.11 measurement messungen rssi signal strength rate," http://www.ibr.cs.tu-bs.de/kb/ wlan-measurements.html.

[4] L.M. Feeney, "Investigating the energy consumption of a wireless network Interface in an ad hoc networking environment," in INFOCOM 2001, 2001, pp. 1548-1557.

[5] Jean-Pierre Ebert, Stephan Aier, Gunnar Kofahl, Alexander Becker, Brian Burns, and Adam Wolisz, "Measurement and simulation of the energy consumption of an wlan interface," Tech. Rep., Technical University of Berlin - TKN, 2002, http: //www.tkn.tu-berlin.de/publications/papers / networking2002\withcovoer.pdf.

[6] K. Mahmud, M. Inoue, H. Murakami, M. Hasegawa, and H. Morikawa, "Energy consumption measurement of wireless interfaces in multi-service user terminals for heterogeneous wireless networks," IEICE TRANS. COMMUN., vol. 88, no. 3, Mar. 2005.
TABLE III

POWER LEVELS USED IN BROAdCAST - 1000 BYTE

\begin{tabular}{lrrr}
\hline state & power value [W] & data rate [Mbps] & loss rate [\%] \\
\hline sending & 1.629 & 5.623 & - \\
receiving @ 3m & 1.375 & 5.379 & 3.328 \\
receiving @ 30m & 1.213 & 5.115 & 8.324 \\
idle @ 3m & 0.979 & - & - \\
idle @ 30m & 0.952 & - & - \\
sleep & 0.04 & - & - \\
\hline
\end{tabular}

